\title{
Correlation between Taylor Model Prediction and Transmission Electron Microscopy-Based Microstructural Investigations of Quasi-In Situ Tensile Deformation of Additively Manufactured FeCo Alloy
}

\author{
Sudipta Pramanik, Lennart Tasche, Kay-Peter Hoyer, and Mirko Schaper
}

\author{
Submitted: 20 May 2021 / Accepted: 11 June 2021 / Published online: 3 August 2021
}

\begin{abstract}
Within this research, the multiscale microstructural evolution before and after the tensile test of a $\mathrm{FeCo}$ alloy is addressed. X-ray $\mu$-computer tomography (CT), electron backscattered diffraction (EBSD), and transmission electron microscopy (TEM) are employed to determine the microstructure on different length scales. Microstructural evolution is studied by performing EBSD of the same area before and after the tensile test. As a result, $\langle 001\rangle\|\mathrm{TD},\langle 011\rangle\| \mathrm{TD}$ are hard orientations and $\langle 111\rangle \| \mathrm{TD}$ is soft orientations for deformation accommodation. It is not possible to predict the deformation of a single grain with the Taylor model. However, the Taylor model accurately predicts the orientation of all grains after deformation. $\{123\}$ $\langle 111\rangle$ is the most active slip system, and $\{112\}\langle 111\rangle$ is the least active slip system. Both EBSD micrographs show grain subdivision after tensile testing. TEM images show the formation of dislocation cells. Correlative HRTEM images show unresolved lattice fringes at dislocation cell boundaries, whereas resolved lattice fringes are observed at dislocation cell interior. Since Schmid's law is unable to predict the deformation behavior of grains, the boundary slip transmission accurately predicts the grain deformation behavior.
\end{abstract}

Keywords EBSD, FeCo alloy, quasi-in situ tensile test, Taylor model, TEM

\section{Introduction}

Soft magnetic materials are important components of electric machine devices requiring high flux density. Among soft magnetic materials, equiatomic FeCo alloys possess the highest saturation magnetization. These alloys also reveal a high Curie temperature and good permeability combined with adequate tensile strength (Ref 1). Nevertheless, the brittle nature of ordered FeCo alloys imparts difficulties in the manufacturing of electric machine components (Ref 1). Additive manufacturing is being identified as a convenient way to produce ductile bulk FeCo alloys in near-net shape.

In the literature, the majority of investigations are related to the magnetic properties of additively manufactured (AM) FeCo and $\mathrm{FeCoV}$ alloys (Ref 2-4). While the magnetic property is a focal point, the deformation behavior is also important in manufacturing the ductile FeCo alloy. In this regard, there are some publications on the deformation behavior of $\mathrm{AM} \mathrm{FeCo}$ alloys (Ref 3,5$)$, in which dislocation slip and subgrain rotation

Supplementary Information The online version contains supplementary material available at https://doi.org/10.1007/s11665-02 1-06065-9.

Sudipta Pramanik, Lennart Tasche, Kay-Peter Hoyer, and Mirko Schaper, Chair of Materials Science, Paderborn University, Mersinweg 7, 33100 Paderborn, Germany. Contact e-mail: pramanik@1wk.upb.de. are the main deformation mechanism. In this regard, the high cooling rate during additive manufacturing leads to atomic disorder (Ref 2, 6, 7). This further increases the ductility. The design of AM part also affects the ductility of the product. The application of additional artificial struts structures during additive manufacturing leads to better mechanical properties compared to sample AM without artificial struts (Ref 3). The struts increase the heat extraction rate leading to a higher cooling rate. This leads to higher disorder and better mechanical properties.

AM FeCo alloys are reported to have high ductility $(\approx 35 \%)$ compared to conventionally processed FeCo alloys (Ref 5). This is due to a multiscale microstructure (fine-scale dislocation structure and voids) showing dislocation accommodation and also impeding dislocation motion (Ref 5). Thus, following the previous study (Ref 5), this paper characterizes the microstructure on multiple length scales (from $\mu \mathrm{m}$ to sub-nm level) to understand the deformation accommodation mechanism of the AM FeCo alloy using a quasi-in situ approach. A detailed multiscale microstructural characterization enables to observe deformation behavior from millimeter to nanometer lengths. Therefore, the internal porosity is studied by x-ray microtomography $(\mu-\mathrm{CT})$ on the millimeter scale. Subsequently, the grain rotation of the same region before and after tensile testing is examined via electron backscattering diffraction (EBSD) at the submicron scale. In this regard, the grain rotation behavior is examined using the Schmid factor and grain boundary slip transmission factor. The grain boundary shape change upon deformation is analyzed using fractal behavior. The Taylor model is used to explain the overall texture of the same sample region before and after the tensile test. Here, the focus is to identify active slip systems and to explain the overall deformation behavior. Further, the evolution of the deformation substructure is addressed by transmission electron microscopy 
(TEM) and correlative high-resolution transmission electron microscopy (HRTEM) on the nanometer level. The dislocation density is also calculated from the deformation substructure with the help of energy-filtered transmission electron microscopy (EFTEM).

\section{Experimental Procedure}

\subsection{Sample Preparation}

Spherical pre-alloyed FeCo powder containing 50 wt.\% Co is produced by gas atomization. The average $\mathrm{d} 50$ (diameter of $50 \%$ particles) is $38 \mu \mathrm{m}$. All FeCo specimens are produced by LBM $\left(\mathrm{SLM} 250^{\mathrm{HL}}\right.$ machine, SLM Solutions Group AG, Lübeck, Germany) applying a $400 \mathrm{~W}$ ytterbium continuous laser with a spot size of $70 \mu \mathrm{m}$. Argon gas is supplied during LBM to prevent oxidation, and the building platform is heated to $200{ }^{\circ} \mathrm{C}$ to reduce internal stress. Based on our previous parameter optimization study (Ref 8), a laser power of $270 \mathrm{~W}$, a scan speed of $700 \mathrm{~mm} / \mathrm{s}$, a hatch distance of $0.11 \mathrm{~mm}$, and a powder layer thickness of $0.05 \mathrm{~mm}$ are set as process parameters.

\subsection{Tensile Testing}

Tensile samples with the gauge section parallel to the build direction (BD) are prepared by LBM. The dimension of the gauge section is $10 \mathrm{~mm}$ (length) $\times 3 \mathrm{~mm}$ (width) $\times 1.5 \mathrm{~mm}$ (thickness). Tensile testing (MTS tabletop system 858) is conducted with the tensile direction (TD) parallel to BD. A strain rate of $10^{-3} \mathrm{~s}^{-1}$ is imposed during tensile testing, and the strain is measured with an extensometer.

\section{$2.3 \mu-C T$}

To determine the internal porosity, $\mu$-CT (Skyscan 1275, Bruker) is performed on the gauge section before the tensile tests. For this, the $\mu$-CT is operated at $100 \mathrm{kV}$ and $10 \mathrm{~W}$ with a tungsten target and copper filter ( $1 \mathrm{~mm})$. During $\mu$-CT, a sample rotation of $360^{\circ}$ is imposed in a step size of $0.2^{\circ}$. A sample volume of $20.2 \mathrm{~mm}^{3}$ is analyzed by $\mu$-CT. For $\mu$-CT data postprocessing, a $7 \mu \mathrm{m}$ voxel size is used.

\subsection{EBSD Sample Preparation and Investigation}

Before performing the tensile tests, all samples are polished using $\mathrm{SiC}$ abrasive paper. The gauge section is further electropolished (Struers Lectropol-5) with an electrolyte containing $590 \mathrm{~mL}$ ethanol, $330 \mathrm{~mL}$ butoxyethanol, and $78 \mathrm{~mL}$ perchloric acid for acquiring EBSD maps. Operating conditions during electropolishing are $20 \mathrm{~V}$ and $1{ }^{\circ} \mathrm{C}$ for $60 \mathrm{~s}$.

Before performing the tensile tests, the gauge sections of the electropolished samples are examined via optical microscopy (Zeiss Axiophot). Furthermore, EBSD maps are captured in the same part of the gauge section before and after the tensile tests. For this, EBSD is performed in a scanning electron microscope (SEM, Zeiss Ultra Plus) equipped with an EBSD detector (EDAX). The SEM is operated at $13.5 \mathrm{~mm}$ working distance and $20 \mathrm{kV}$ accelerating voltage; the EBSD maps are collected at $200 \mathrm{~nm}$ step size and $77 \mathrm{pA}$ probe current.

\subsection{EBSD Data Analysis}

The EBSD file is exported from TSL OIM 7.1 software to MTEX 5.4.0 software (Ref 9). The EBSD data analysis is done using MTEX 5.4.0 (Ref 9). A misorientation angle larger than $15^{\circ}$ is defined as a high angle grain boundary. Finally, the inverse pole figure (IPF) map along TD is plotted. Based on this, the Schmid factor (SF) of grains is calculated considering $\{110\}\langle 111\rangle,\{112\}\langle 111\rangle$ and $\{123\}\langle 111\rangle$ slip systems. Moreover, the Taylor model is applied to predict the deformation behavior during tensile testing. A strain tensor $[1,0,0 ; 0$, $-0.5,0 ; 0,0,-0.5]$ is imposed for calculation in the Taylor model.

The grain boundary shape before and after the tensile test is characterized by the fractal number. The fractal number is calculated using the multiple iterations box-counting methods. In the box-counting method, the boxes of size $r$ are drawn on the micrograph and the number of boxes containing the grain boundaries is counted. The size of boxes is changed in each iteration, and the number of boxes containing the grain boundaries is counted again. The fractal number is calculated using Eq 1:

$N=r^{-D}$

where $N$ is the number of boxes, $r$ is the size of the box, and $D$ is the fractal number (Ref 10).

\subsection{TEM Sample Preparation and Investigation}

After the tensile tests, the gauge section of each sample is cut applying the Struers Secotom-5. For TEM, a disk with a diameter of $3 \mathrm{~mm}$ is prepared and subsequently polished to a thickness of $\approx 100 \mu \mathrm{m}$. For preparing the TEM sample, electropolishing is done using a $5 \%$ perchloric acid-ethanol solution in Struers Tenupol-5 at a voltage of $21 \mathrm{~V}$, a current of $30 \mathrm{~mA}$, and a temperature of $-21{ }^{\circ} \mathrm{C}$.

Subsequently, TEM is performed using the JEOL JEMARM 200F which is equipped with a cold field emission gun and a CEOS hexapole $\mathrm{C}_{\mathrm{S}}$ corrector. An emission current of 15 $\mu \mathrm{A}$ is used for sample imaging. A double tilt sample holder is used during all TEM investigations, and HRTEM images are also acquired.

Additionally, EFTEM images are captured to measure the average sample thickness. For capturing the EFTEM images, an energy filter of width $10 \mathrm{eV}$ is selected. An EFTEM image is taken with the energy filter placed around the zero-loss peak. Another EFTEM image is taken without any image filter. The sample thickness $(t)$ is calculated using Eq 2:

$t=\lambda \ln \left(I / I_{o}\right)$

where $\lambda$ is mean free path, $I_{o}$ is the intensity with the energy filter, and $I$ is the intensity without the energy filter. The mean free path is calculated from the chemical composition using the Iakoubovskii model (Ref 11). The model is implemented using the Digital Micrograph script (Ref 12).

To calculate the dislocation density, lines are drawn on each TEM micrograph. Afterward, the dislocation density is calculated based on $\mathrm{Eq} 3$ :

$\rho=\frac{2 N}{L t}$

(Eq 3) 
where $\rho$ is the dislocation density $\left(\mathrm{m}^{-2}\right), N$ is the number of intersection points, $L$ is the total length of all the lines (m), and $t$ is the sample thickness (m) (Ref 13).

\section{Results}

\subsection{Optical Microscopy and $\mu$-CT}

Figure 1(a) shows a schematic of a tensile sample. The gauge section is highlighted in yellow. The optical micrograph of the representative gauge section in Fig. 1(b) proves an almost defect-free sample surface, which is supported by the $\mu$ CT image in Fig. 1(c) showing the gauge section of the same specimen. Only little internal porosity is observed. However, the total porosity is determined to be $0.1 \%$. Figure $1(\mathrm{~d})$ shows a plot of the pore diameter versus sphericity of pores revealed from the $\mu$-CT data. Based on the literature (Ref 14), the pores are classified into gas porosity or incomplete fusion porosity. Most of the pores are spherical (sphericity $\approx 0.9$ ) and are formed due to gas entrapment during LBM. During LBM, the melt pool is at a higher temperature resulting in high solubility of gases. Due to very fast cooling during LBM, there is insufficient time for the dissolved gases to escape the melt pool (Ref 15). This results in gas entrapment.

\subsection{Tensile Test}

Figure 2 summarizes the engineering stress-strain curve and strain hardening rate curve for the FeCo specimen. Based on the engineering stress-strain curve in Fig. 2(a), the yield strength is $315 \mathrm{MPa}$, the ultimate tensile strength (UTS) is 738 $\mathrm{MPa}$, and the total elongation is $34 \%$. The engineering stressstrain curve shows only a little elastic linear behavior, followed by a sharp increase in stress until UTS. Subsequently, after UTS a gradual decrease in stress is observed due to strain localization. The strain hardening rate curve calculated from the true stress-strain curve is shown in Fig. 2(b). Stage 1 is characterized by a rapid decrease in strain hardening rate due to the onset of dynamic recovery. Stage 2 shows a slower decrease in strain hardening rate due to the onset of slip-on different slip (a)
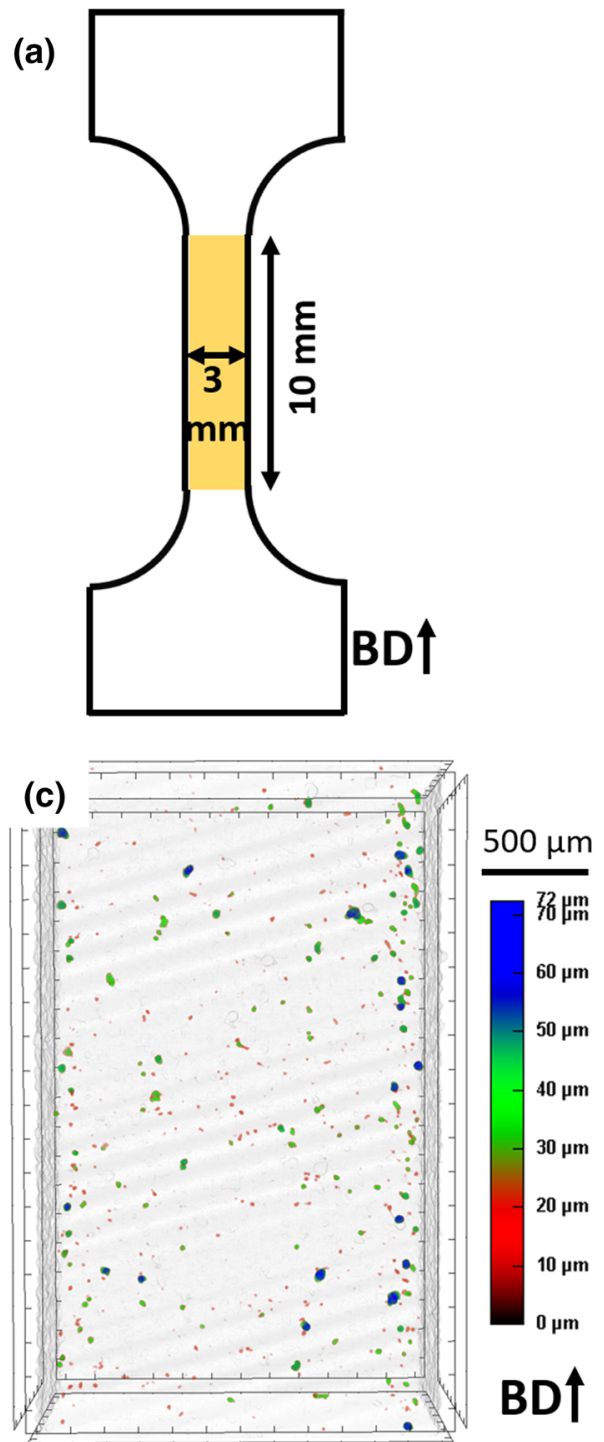

(b)

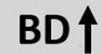

$\overline{40 \mu \mathrm{m}}$

(d)

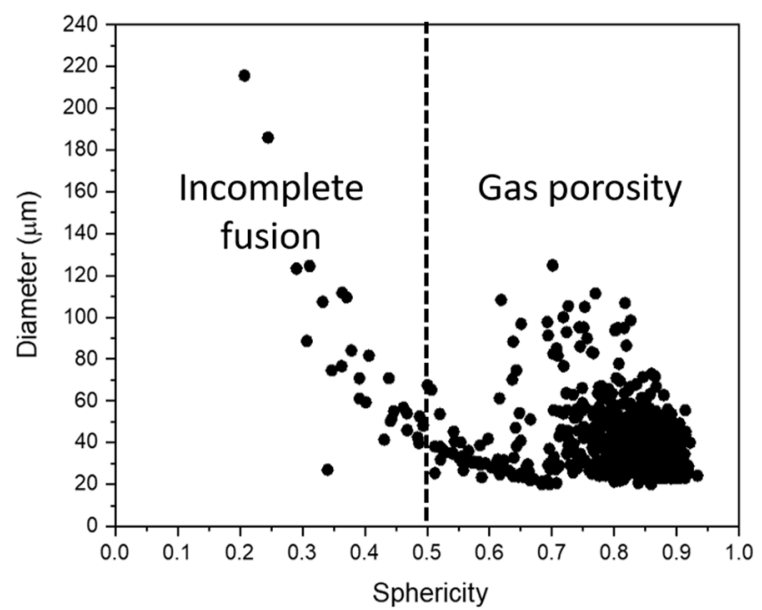

Fig. 1 (a) Schematic of the tensile sample, (b) optical micrograph, (c) $\mu$-CT of the gauge section, and (d) correlation of the pore diameter and the sphericity of pores. BD, build direction. The gauge section is highlighted in (a). The color bar in (b) shows the distribution of the pore sizes. 

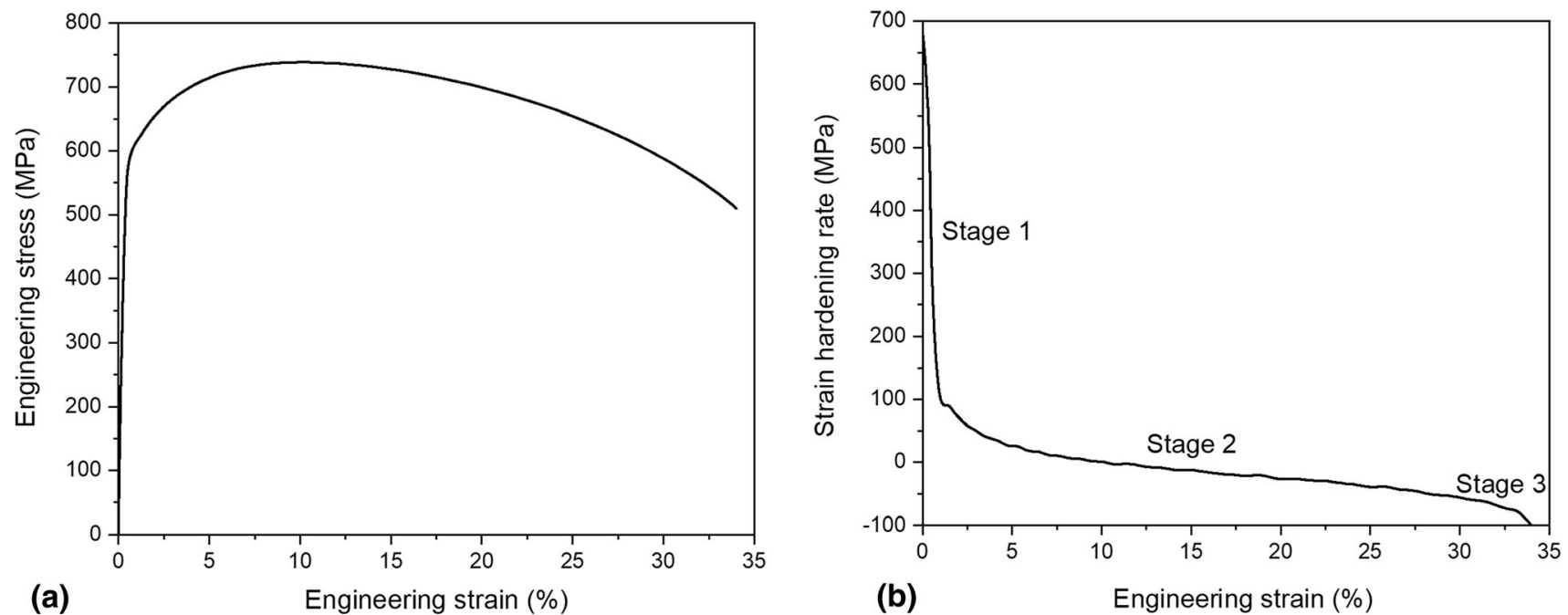

Fig. 2 (a) Engineering stress-strain curve and (b) strain hardening rate curve of FeCo sample

systems. Stage 3 which represents the onset of failure due to strain localization is not shown here.

\subsection{EBSD Investigations}

Figure 3(a) and (b) shows the EBSD map of the same area before and after the tensile test. The microstructures in Fig. 3 (a) and (b) are acquired from an area away from the necking region. Almost all grains are elongated along with the $\mathrm{BD} / \mathrm{TD}$. The average grain size before and after tensile testing is $3.7 \mu \mathrm{m} \pm 2.9 \mu \mathrm{m}$ and $3.7 \mu \mathrm{m} \pm 3.1 \mu \mathrm{m}$, respectively. Upon plotting the grain orientations on IPF (Supplementary Figure S1), the [111]||TD orientations have the lowest SF, whereas [001]||TD orientations exhibit the highest SF. Therefore, [001]||TD orientated grains are anticipated to undergo deformation. However, experimental data show that [001]||TD and [011]||TD oriented grains are hard orientation. These orientations do not undergo much rotation and are resistant to deformation accommodation.

During fractal analysis, a linear relationship between the box size and the number of boxes is observed (Supplementary Figure S2). This is due to the fractal nature of the microstructure. From the slope of the curve, the fractal number before and after tensile testing is 1.84 indicating no change in fractal behavior. As there is no change in the fractal behavior, no significant grain boundary shape change is expected for the overall microstructure. But there may be grain boundary shape change on a local scale.

Figure 3(a) displays a [001]||TD oriented "grain 1". Correspondingly, Fig. 3(c) indicates "grain 1" to have a high $\mathrm{SF}$ value $(>0.48)$. However, no significant grain rotation is observed for "grain 1" in Fig. 3(b). Therefore, Schmid's law is inapplicable to explain the deformation of this grain. Figure 3(a) also highlights "grain $2 "$ with a $\left[\begin{array}{ll}1 & \overline{24}\end{array}\right] \mid$ TD orientation. According to Fig. 3(c), this should have a high SF value ( $>$ 0.46), too. "Grain 2" undergoes significant rotation upon deformation. Therefore, Schmid's law is applicable to explain its deformation. Consequently, Schmid's law is capable to explain the deformation behavior of only some specific grains (here: grain 2).

Also, Fig. 3(d) presents a map with a slip transmission factor (Ref 16) across boundaries. Here, the slip transmission factor quantifies grain boundary ability to transmit slip between two neighboring grains. The slip transmission factor measures the relative orientation of slip planes and slip directions for slip transmission between two neighboring grains. A slip transmission factor value equal to one means no grain boundary resistance to slip transmission. Slip transmission factor (Ref 17) is calculated using $\mathrm{Eq}$ 4:

$m^{\prime}=\cos \phi \cdot \cos \kappa$

where $\phi$ is the angle between slip plane normals, $\kappa$ is the angle between slip directions, and $m^{\prime}$ is the slip transmission factor.

In Fig. 3(d), "grain 1" has boundaries with low slip transmission $(\approx 0.2)$, whereas "grain 2 " has boundaries with high slip transmission $(\approx 0.9)$. Hence, "grain 2 " is more susceptible to deformation accommodation compared to "grain 1". Based on this, boundary slip transmission is capable to explain the deformation behavior of both grains.

\subsection{Taylor Model Simulation}

It is well known that the deformation behavior of grain is influenced by its neighboring grains. To restrict the number of neighboring grains, the highlighted grain (dotted rectangle in Fig. 3(a) and (b)) is chosen for local analysis. This grain is completely circumscribed within a single grain. Figure 4(a) depicts the EBSD map of the highlighted grain (dotted rectangle in Fig. 3(a) and (b)) before and after tensile testing. A fragmentation of grain orientations (IPF blue dots, Fig. 4(a)) is observed after deformation. Here, the Taylor model is applied to predict grain orientation. As the EBSD observation area is away from the neck section, the deformation level is assumed to be a uniform elongation strain for the Taylor model. It is stated that the Taylor model does not predict the orientation of the individual grain accurately. According to the IPF in Fig. 4(a), a rotation of $\approx 11^{\circ}$ is expected [00 1 ] between the initial orientation and the prediction based on the Taylor model. However, after the tensile test a rotation of $\approx 6^{\circ}[\overline{132}]$ occurred.

Applying the Taylor model to predict the global orientation of all grains in Fig. 3(a) leads to the results summarized in Fig. 4(b). The superimposed IPF in Fig. 4(b) presents all grain orientations before (black dots) and after tensile tests (red dots) as well as the prediction of the Taylor model (blue dots). 

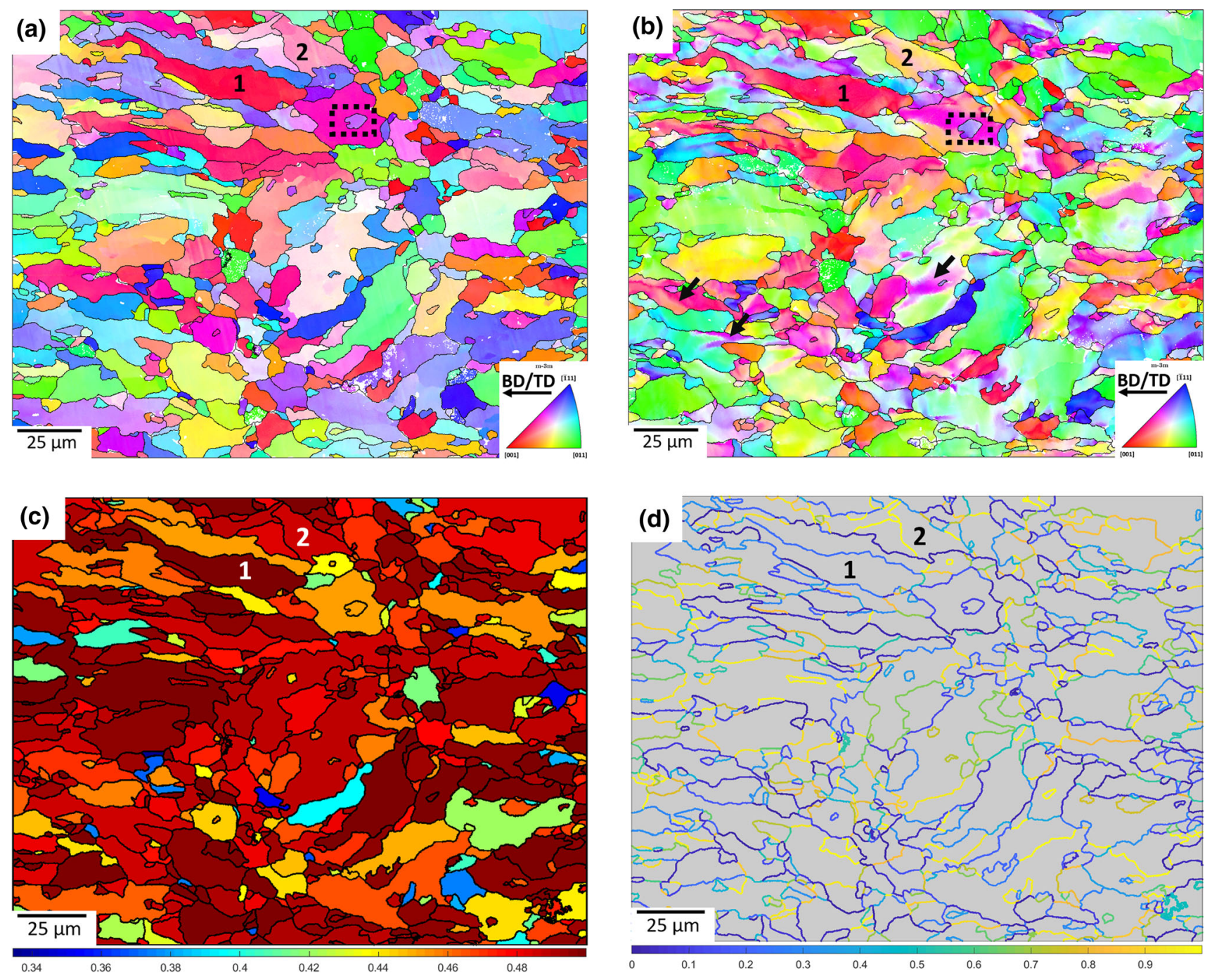

Fig. 3 EBSD map of the gauge section (a) before and (b) after performing tensile tests. (c) Schmid's factor map of (a). (d) The boundary slip transmission map of (a). (BD, build direction; $\mathrm{TD}$, tensile direction. Black arrows highlight grain fragmentation.)

Besides, the contour IPF plot shows a clustering of orientations to [056]||TD according to the Taylor model (Supplementary Figure S3). From the experiments, a formation of strong [146]||TD and [023]||TD orientations is stated (Supplementary Figure S4). These orientations are close to [056||TD which is predicted by the Taylor model. A $\langle 101\rangle$ texture is reported to develop during tensile testing of bcc crystals (Ref 18) and also for FeCo alloy (Ref 5).

\subsection{TEM Investigations}

Figure 5 shows TEM and HRTEM images from the gauge section after deformation. In Fig. 5(a), yellow arrows indicate grain boundaries. The formation of dislocation cells (red arrows) is observed. The dislocation cell boundaries are not sharp. The size of the dislocation cells is not uniform and varies between $\approx 130$ and $\approx 360 \mathrm{~nm}$. The magnified images of dislocation cells from a different region are shown in Supplementary Figure S5. The size of the dislocation cells here is between $\approx 140 \mathrm{~nm}$ to $\approx 400 \mathrm{~nm}$. This supports the results from the previous EBSD measurements regarding grain fragmentation (Fig. 3b). The local average thickness of the TEM sample is calculated as $\approx 51 \mathrm{~nm}$ (Supplementary Figure S6). The dislocation density in Fig. 5(a) calculated according to Eq 2 is $8.5 \times 10^{13} \mathrm{~m}^{-2}$. In this regard, our previous study shows a dislocation density in the as-built FeCo alloy sample to be $2.2 \times 10^{13} \mathrm{~m}^{-2}$ (Ref 8). Thus, an increase in dislocation density is observed upon the tensile test. Deformation-induced substructure formation is not reported for $\mathrm{FeCo}$ alloy in previous literature. Previous studies have reported the development of subgrains in FeCo alloy upon cold rolling and annealing. In this regard, for FeCo1.8V0.3 Nb alloy, the size of the subgrains is reported to be $100 \mathrm{~nm}$ and $150 \mathrm{~nm}$ for different annealing treatments (Ref 19). However, for FeCo2V alloy the subgrain size is $1 \mu \mathrm{m}$ after annealing (Ref 20).

Figure 5(b), (c) and (d) represents the correlative HRTEM images presenting the local lattice structure. Figure 5(b) is taken from the interior of a dislocation cell (Fig. 5a). Figure 5(c) and (d) is taken from a region close to a dislocation cell boundary (Fig. 5a). The inset fast Fourier transform (FFT) pattern in Fig. 5(b) shows FeCo [012] zone axis. The lattice spacing of (121) planes measured from the FFT pattern is $\approx 0.11 \mathrm{~nm}$. Figure 5(c) shows the lattice fringes due to (121) planes with a spacing of $\approx 0.11 \mathrm{~nm}$. Due to the presence of lattice strain near the dislocation cell boundary, the contrast from the lattice fringes is not clear as highlighted by white arrows (Fig. 5c). 


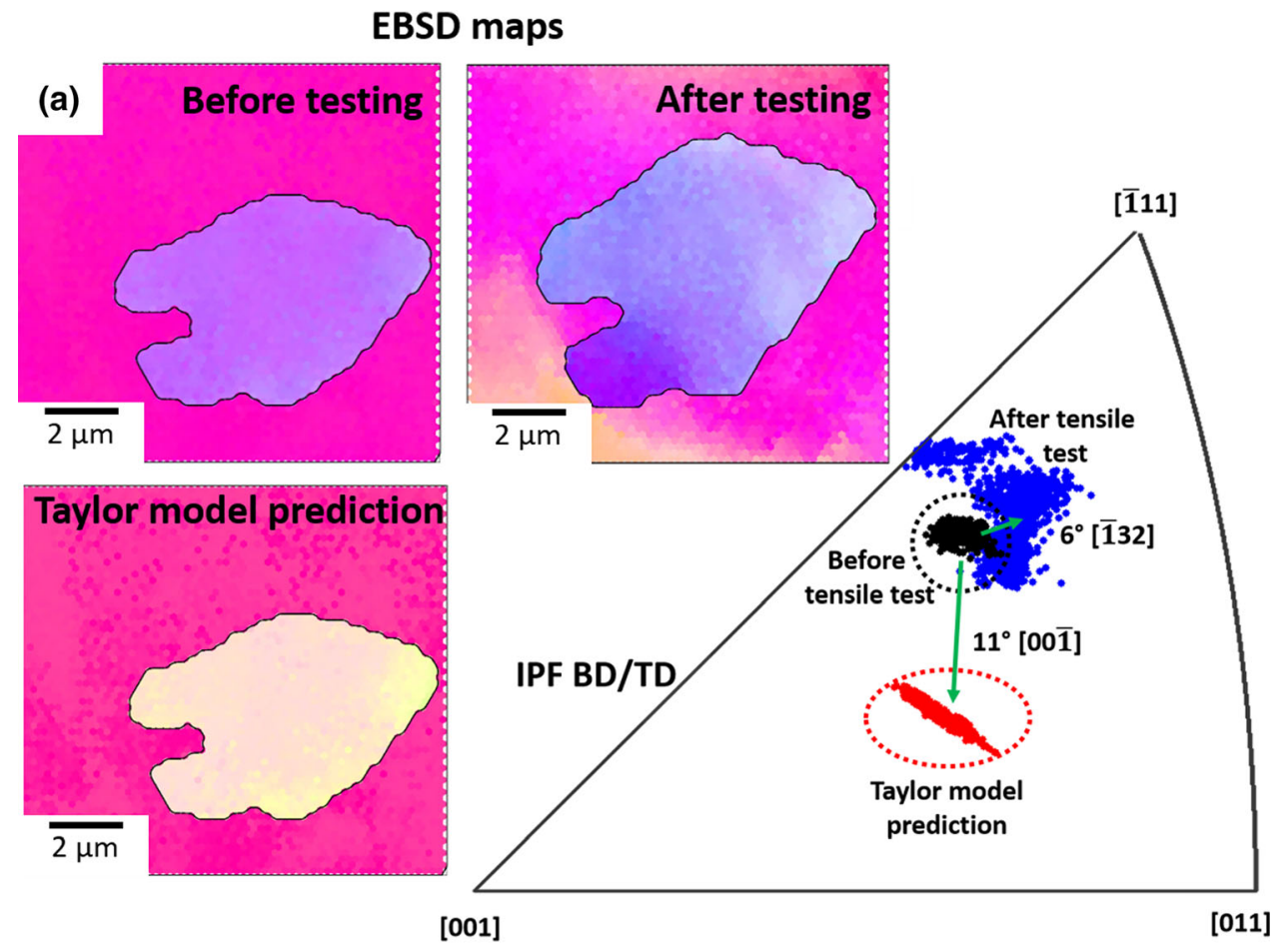

(b)

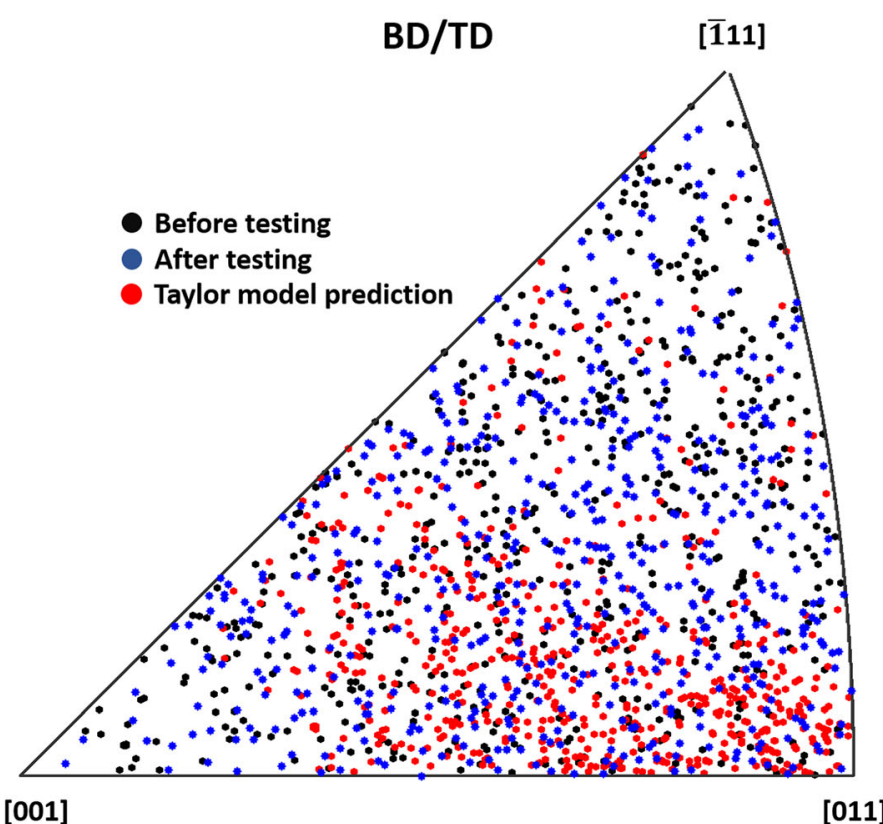

Fig. 4 (a) Section of the EBSD map and inverse pole figure of the single grain before and after deformation and (b) inverse pole figure of all grains before and after deformation as well as the prediction based on the Taylor model. BD, build direction; TD, tensile direction

The lattice fringes are due to (110) planes with a spacing of $\approx 0.20 \mathrm{~nm}$ (Supplementary Figure S7). The inset FFT pattern in Fig. 5(d) shows the FeCo [ 113$]$ zone axis. The presence of areas with unresolved lattice fringes is highlighted by white arrows (Fig. 5d). These areas are due to lattice strain or lens aberrations. Lattice strains are attributed to the presence of dislocations due to grain subdivision. Extensively increased dislocation density, grain rotation, and subgrain formation are observed in a tensile tested FeCo alloy sample (Ref 5).

\section{Discussions}

The inability of the Taylor model to accurately predict the orientations of one single grain upon deformation is due to its assumption. The main assumption is that each grain experiences the same amount of external macroscopic strain. This may not be true on a microscopic level of a single grain. Furthermore, interaction effects between grains are disregarded. 

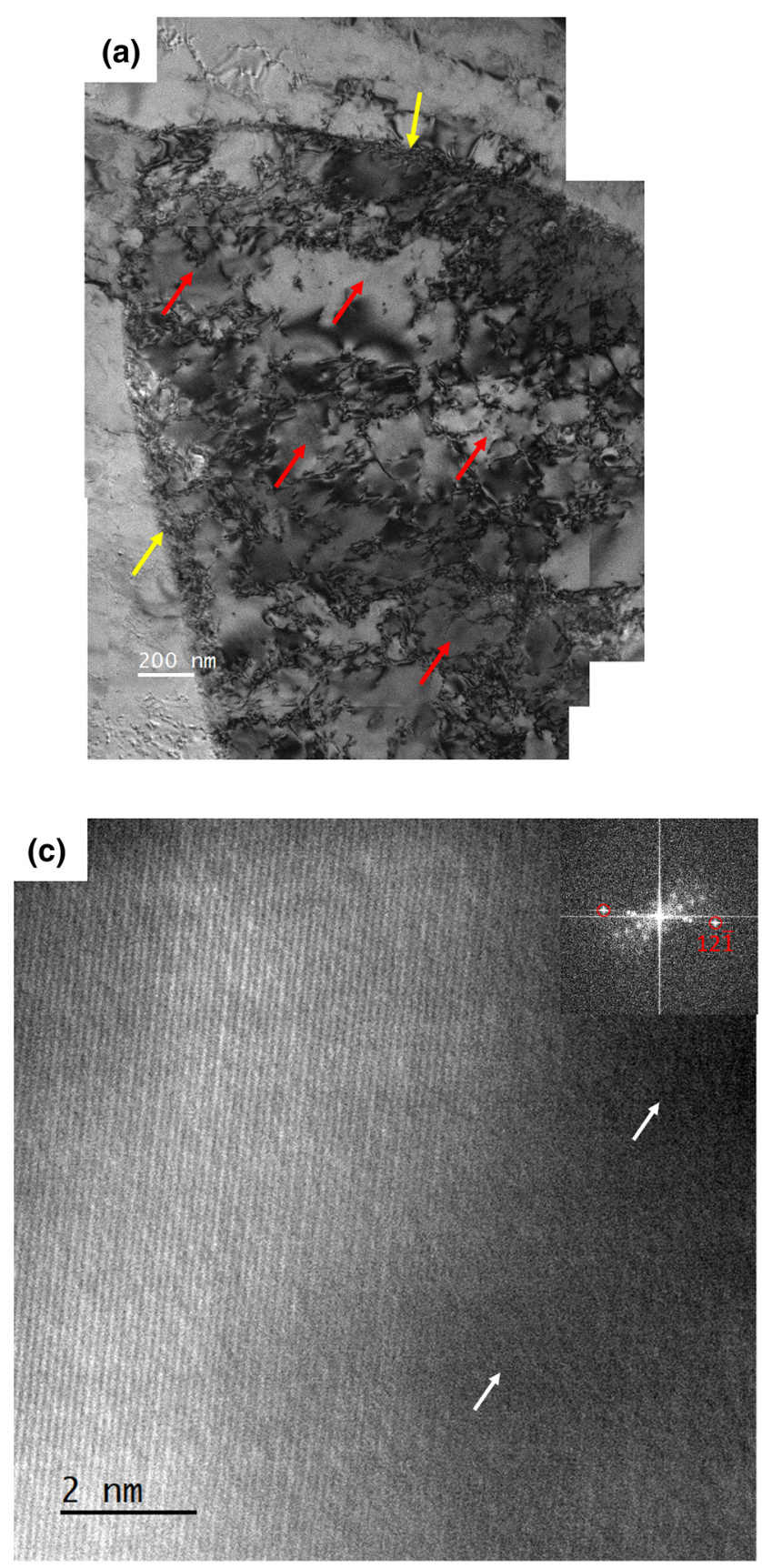

(b)

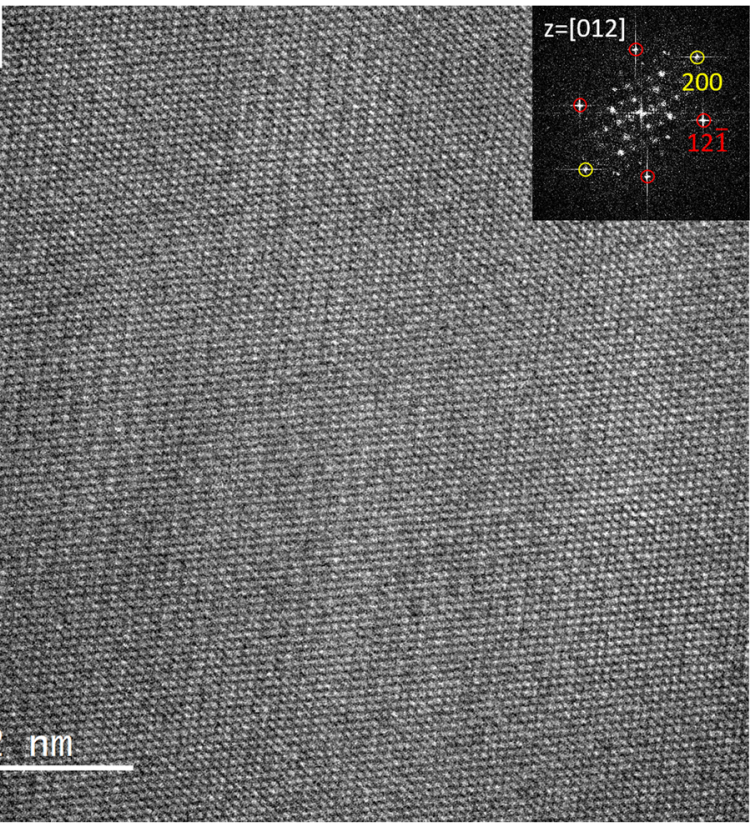

(d)

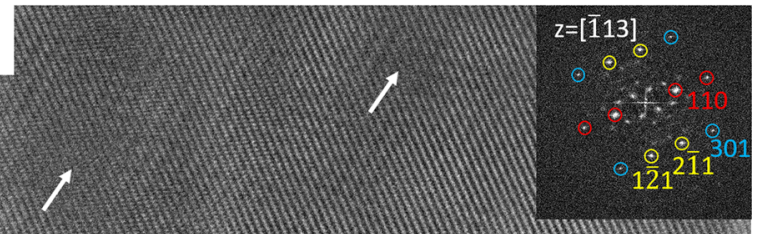

Fig. 5 (a) TEM and (b), (c), (d) HRTEM micrographs of the gauge section. In (a) yellow and red arrows indicate grain boundaries and subgrains, respectively. In (c), (d) the white arrows show unresolved lattice fringes.

This leads to inaccurate predictions of the orientations in the deformed state. However, the Taylor model predicts the texture evolution of all grains in the global microstructure accurately. Based on the coefficient of the Taylor model, the activity of three slip systems of the FeCo alloy is presented in Table 1. It is observed that all three slip systems are activated in varying amounts. $\{123\}\langle 111\rangle$ is the most active and $\{112\}\langle 111\rangle$ the least active slip system. In this regard, Fig. 6 shows the sample surface of the tensile deformed sample. The presence of slip bands and wavy slip lines is seen. This is an indication of cross slip due to the activation of multiple slip systems. Moreover, upon tensile deformation orientation change within grains is highlighted by black arrows in Fig. 3(b). This is due to grain
Table 1 Activity of slip systems of FeCo alloy based on the deformation in tensile tests

\begin{tabular}{lc}
\hline Slip system & Relative activity \\
\hline$\{110\}\langle 111\rangle$ & 7.6 \\
$\{112\}\langle 111\rangle$ & 5.6 \\
$\{123\}\langle 111\rangle$ & 14.2 \\
\hline
\end{tabular}

fragmentation due to the action of different slip system on different parts of the grain. Qualitatively, stage 2 of the strain hardening curve also indicates the activation of multiple slip systems. The high dislocation density may be also due to 

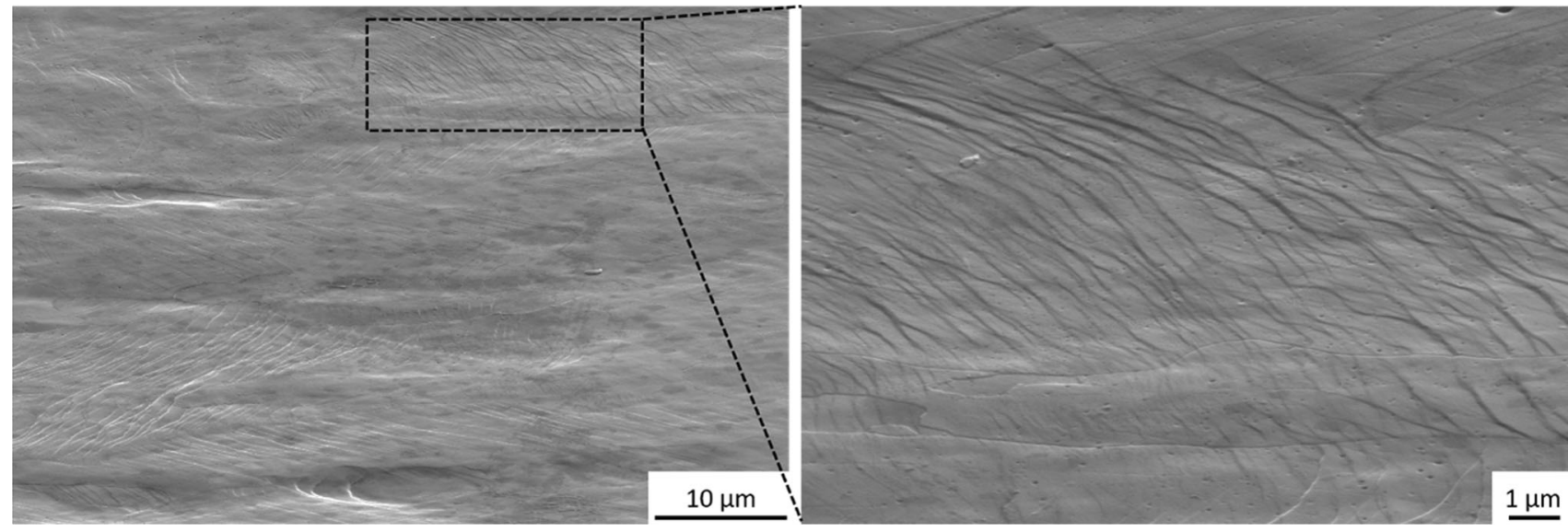

Fig. 6 SEM micrograph of the deformed sample showing wavy slip lines

multiple slip system activation. Previous studies reported only the activation of the $\{110\}\langle 111\rangle$ slip system (Ref 1). It is stated that the $\{110\}\langle 111\rangle$ slip system provides five independent slip systems and thereby satisfies the von Mises criteria for polycrystalline plasticity. However, in the current analysis, the five independent slip systems are not activated only by the $\{110\}\langle 111\rangle$ slip system. Therefore, the activation of more slip systems is required during the deformation.

\section{Conclusions}

Based on the study, the following conclusions can be drawn:

1. Grain fragmentation is observed after tensile testing due to the activation of multiple slip systems. Grains with [001]||TD and [011]||TD orientations are observed to be resistant to deformation accommodation. No significant change in grain boundary shape (fractal number) is observed before and after the tensile test.

2. Schmid's law is not applicable to explain the deformation behavior of all grain orientations. However, the grain boundary slip transmission is suitable to explain the global deformation behavior of all grain orientations.

3. The Taylor model does not accurately predict the orientation for one single grain upon deformation. However, the model predicts the orientation of all grains globally upon deformation with high accuracy. The $\{123\}\langle 111\rangle$ slip system is the most activated one, whereas the $\{112\}\langle 111\rangle$ slip system is the least activated during tensile testing.

4. Based on the EBSD micrographs, a grain subdivision after tensile testing is suggested. Here, TEM micrographs support the occurrence of dislocation cells. The dislocation density in the deformed sample is $8.5 \times 10^{13} \mathrm{~m}^{-2}$. Correlative HRTEM images show the unresolved lattice fringes near the dislocation cell boundary and resolved lattice fringes in the dislocation cell interior.

\section{Acknowledgments}

The authors would like to thank Anja Puda and Thomas Janzen for their support in sample preparation and execution of the hardness tests and EBSD analysis. Dr.-Ing Anatoli Andreiev and Dennis Milaege are acknowledged for performing $\mu$-CT measurements and tensile tests.

\section{Funding}

Open Access funding enabled and organized by Projekt DEAL.

Open Access This article is licensed under a Creative Commons Attribution 4.0 International License, which permits use, sharing, adaptation, distribution and reproduction in any medium or format, as long as you give appropriate credit to the original author(s) and the source, provide a link to the Creative Commons licence, and indicate if changes were made. The images or other third party material in this article are included in the article's Creative Commons licence, unless indicated otherwise in a credit line to the material. If material is not included in the article's Creative Commons licence and your intended use is not permitted by statutory regulation or exceeds the permitted use, you will need to obtain permission directly from the copyright holder. To view a copy of this licence, visit http://creativecommons.org/licenses/by/4.0/.

\section{Data Availability}

The raw/processed data required to reproduce these findings cannot be shared at this time as the data also form part of an ongoing study.

\section{References}

1. R.S. Sundar and S.C. Deevi, Soft Magnetic FeCo Alloys: Alloy Development, Processing, and Properties, Int. Mater. Rev., 2005, 50(3), p 157-192. https://doi.org/10.1179/174328005X14339

2. A.B. Kustas, D.F. Susan, K.L. Johnson, S.R. Whetten, M.A. Rodriguez, D.J. Dagel, J.R. Michael, D.M. Keicher and N. Argibay, Characterization of the Fe-Co-1.5V Soft Ferromagnetic Alloy Processed by Laser Engineered Net Shaping (LENS), Addit. Manuf., 2018, 21, p $41-52$

3. T.F. Babuska, K.L. Johnson, T. Verdonik, S.R. Subia, B.A. Krick, D.F. Susan and A.B. Kustas, An Additive Manufacturing Design Approach to Achieving High Strength and Ductility in Traditionally Brittle 
Alloys via Laser Powder Bed Fusion, Addit. Manuf., 2020, 34, p 101187

4. V. Chaudhary, N.M. Sai Kiran Kumar Yadav, S.A. Mantri, S. Dasari, A. Jagetia, R.V. Ramanujan and R. Banerjee, Additive Manufacturing of Functionally Graded Co-Fe and Ni-Fe Magnetic Materials, J. Alloys Compd., 2020, 823, p 153817

5. T.F. Babuska, M.A. Wilson, K.L. Johnson, S.R. Whetten, J.F. Curry, J.M. Rodelas, C. Atkinson, P. Lu, M. Chandross, B.A. Krick, J.R. Michael, N. Argibay, D.F. Susan and A.B. Kustas, Achieving High Strength and Ductility in Traditionally Brittle Soft Magnetic Intermetallics via Additive Manufacturing, Acta Mater, 2019, 180, p 149157

6. A.B. Kustas, C.M. Fancher, S.R. Whetten, D.J. Dagel, J.R. Michael and D.F. Susan, Controlling the Extent of Atomic Ordering in Intermetallic Alloys through Additive Manufacturing, Addit. Manuf., 2019, 28, p 772-780

7. D.W. Clegg and R.A. Buckley, The Disorder $\rightarrow$ Order Transformation in Iron-Cobalt-Based Alloys, Met. Sci. J., 1973, 7(1), p 48-54. h ttps://doi.org/10.1179/030634573790445541

8. S. Pramanik, L. Tasche, K.-P. Hoyer, and M. Schaper, Investigating the Microstructure of an Additively Manufactured FeCo Alloy: An Electron Microscopy Study, Addit. Manu., 2021, 46, P 102087. http s://doi.org/10.1016/j.addma.2021.102087

9. R. Hielscher and H. Schaeben, A Novel Pole Figure Inversion Method: Specification of the MTEX Algorithm, J. Appl. Crystallogr., 2008, 41(6), p 1024-1037. https://doi.org/10.1107/S0021889808030112

10. S. Kobayashi, R. Kobayashi and T. Watanabe, Control of Grain Boundary Connectivity Based on Fractal Analysis for Improvement of Intergranular Corrosion Resistance in SUS316L Austenitic Stainless Steel, Acta Mater, 2016, 102, p 397-405

11. K. Iakoubovskii, K. Mitsuishi, Y. Nakayama and K. Furuya, Thickness Measurements with Electron Energy Loss Spectroscopy, Microsc. Res. Tech., 2008, 71(8), p 626
12. D.R.G. Mitchell and B. Schaffer, Scripting-Customised Microscopy Tools for Digital Micrograph ${ }^{\mathrm{TM}}$, Ultramicroscopy, 2005, 103(4), p 319-332

13. P.B. Hirsch, Electron Microscopy of Thin Crystals, Butterworths, 1965

14. C.A. Biffi, J. Fiocchi, F. Valenza, P. Bassani and A. Tuissi, Selective Laser Melting of NiTi Shape Memory Alloy: Processability, Microstructure, and Superelasticity, Shape Mem. Superelast., 2020, 6(3), p 342-353. https://doi.org/10.1007/s40830-020-00298-8

15. B. Zhang, Y. Li and Q. Bai, Defect Formation Mechanisms in Selective Laser Melting: A Review, Chinese J. Mech. Eng. (English Edition), 2017 https://doi.org/10.1007/s10033-017-0121-5

16. D. Mercier, C. Zambaldi and T.R. Bieler, A Matlab Toolbox to Analyze Slip Transfer through Grain Boundaries, IOP Conf. Ser. Mater. Sci. Eng., 2015 https://doi.org/10.1088/1757-899X/82/1/012090

17. J. Luster and M.A. Morris, Compatibility of Deformation in Two-Phase Ti-Al Alloys: Dependence on Microstructure and Orientation Relationships, Metall. Mater. Trans. A, 1995, 26(7), p 1745-1756. https://d oi.org/10.1007/BF02670762

18. M. Knezevic, M. Zecevic, I.J. Beyerlein, A. Bhattacharyya and R.J. McCabe, Predicting Texture Evolution in Ta and Ta-10W Alloys Using Polycrystal Plasticity, JOM Miner. Met. Mater. Soc., 2015, 67(11), p 2670-2674. https://doi.org/10.1007/s11837-015-1613-3

19. C.D. Pitt and R.D. Rawlings, Microstructure of Fe-Co-2V and Fe-CoV-Ni Alloys Containing 1-8-7-4 Wt-\%Ni, Met. Sci., 1981, 15(8), p 369-376. https://doi.org/10.1179/030634581790426912

20. A. Duckham, D.Z. Zhang, D. Liang, V. Luzin, R.C. Cammarata, R.L. Leheny, C.L. Chien and T.P. Weihs, Temperature Dependent Mechanical Properties of Ultra-Fine Grained FeCo-2V, Acta Mater., 2003, 51(14), p 4083-4093

Publisher's Note Springer Nature remains neutral with regard to jurisdictional claims in published maps and institutional affiliations. 